\title{
Ultrafast Separation of Photodoped Carriers in Mott Antiferromagnets
}

\author{
Martin Eckstein ${ }^{1, *}$ and Philipp Werner ${ }^{2}$ \\ ${ }^{1}$ Max Planck Research Department for Structural Dynamics, University of Hamburg-CFEL, 22761 Hamburg, Germany \\ ${ }^{2}$ Department of Physics, University of Fribourg, 1700 Fribourg, Switzerland
}

(Received 6 March 2014; published 13 August 2014)

\begin{abstract}
We use inhomogeneous nonequilibrium dynamical mean-field theory to investigate the spreading of photoexcited carriers in Mott insulating heterostructures with strong internal fields. Antiferromagnetic correlations are found to affect the carrier dynamics in a crucial manner: An antiferromagnetic spin background can absorb energy from photoexcited carriers on an ultrafast time scale, thus enabling fast transport between different layers and the separation of electron and holelike carriers, whereas in the paramagnetic state, carriers become localized in strong fields. This interplay between charge and spin degrees of freedom can be exploited to control the functionality of devices based on Mott insulating heterostructures with polar layers, e.g., for photovoltaic applications.
\end{abstract}

DOI: 10.1103/PhysRevLett.113.076405

PACS numbers: $71.10 . \mathrm{Fd}$

Heterostructures and interfaces of transition-metal oxides [1-3] allow us to exploit correlation effects on atomic length scales for the purpose of material design, while femtosecond laser pulses can probe and manipulate the evolution of complex states of matter such as superconducting [4] or magnetically ordered phases [5] on microscopic time scales. Combining ideas and techniques from both fields [6] promises an unprecedented level of control over complex dynamical processes. In this Letter we analyze, in particular, the intriguing nonlinear carrier dynamics in antiferromagnetic Mott-insulating heterostructures which results from the interplay of spin and charge degrees of freedom on femtosecond time scales. Recent theoretical work on nonlinear transport in correlated systems [7-10] has revealed a somewhat counterintuitive effect of dissipation on the carrier dynamics in strong fields. In a closed system, particles remain localized in a potential energy gradient because the electronic bandwidth limits their kinetic energy. Transport is thus enhanced, rather than hampered, by dissipation and the dominant scattering channel, such as phonons or magnons, defines the maximum possible current $[7,8]$. Related nonlinearities can be expected to affect the transport properties of heterostructures with polar layers, which can build up internal fields of order $0.1 \mathrm{~V}$ per lattice spacing $[11,12]$.

An interesting device, where the carrier separation by strong internal fields plays an important role, is the Mott insulating solar cell proposed in Ref. [13]. Its efficient operation is possible only if one can beat the rapid recombination of photoexcited carriers, which happens on much shorter (picosecond) time scales in Mott systems than in semiconductors $[14,15]$. As we will demonstrate, an ultrafast separation of photoinduced carriers is indeed possible in antiferromagnetically ordered materials, where it relies on the capability of the spin sector to act as an "energy bank," which can absorb and store energy on femtosecond time scales, before slowly dissipating it to the lattice and/or leads in a manner which is independent of the dynamics of the photodoped carriers. In a spin-disordered state, on the other hand, the estimated carrier separation times become comparable to typical carrier recombination times in Mott insulators, even for fields approaching the electric breakdown limit of the device. Our results also suggest a way to use spatial inhomogeneities to explore the ultrafast scattering between spins and carriers, complementary to optical spectroscopy [16].

We demonstrate the nontrivial effect of the spin background on the carrier dynamics by considering a model consisting of six layers of a three-dimensional Mott insulator with simple cubic lattice structure, coupled to metallic leads [Fig. 1(d)]. The insulator is described by a Hubbard Hamiltonian,

$$
\begin{aligned}
H= & \sum_{z,\langle\boldsymbol{i}\rangle, \sigma} t_{i \boldsymbol{j}}^{\|} c_{i, z, \sigma}^{\dagger} c_{\boldsymbol{j}, z, \sigma}+\sum_{\left\langle z, z^{\prime}\right\rangle, \boldsymbol{i}, \sigma} t_{z, z^{\prime}}^{\perp} c_{\boldsymbol{i}, z, \sigma}^{\dagger} c_{i, z^{\prime}, \sigma} \\
& +U \sum_{\boldsymbol{j}, z} n_{\boldsymbol{j}, z, \uparrow} n_{\boldsymbol{j}, z, \downarrow}-\Delta E \sum_{\boldsymbol{j}, z, \sigma}(z-3.5) n_{\boldsymbol{j}, z, \sigma},
\end{aligned}
$$

with local Coulomb repulsion $U$, nearest-neighbor intralayer hopping $t_{i j}^{\|}$, and interlayer hopping $t_{z, z^{\prime}}^{\perp} ; z=1, \ldots, 6$ denotes the layer, and $i$ is the lattice coordinate within the infinitely extended $x-y$ plane. A static potential energy difference $\Delta E$ between the layers captures the effect of internal fields in the polar structure.

Methods.-To solve the electron dynamics, we use nonequilibrium dynamical mean-field theory (DMFT) $[17,18]$. DMFT maps a correlated lattice problem onto a self-consistently determined impurity model [19]. The main approximation in this formalism is the assumption of a spatially local self-energy. While spatial correlations are treated at a mean-field level, time-dependent fluctuations can be captured accurately. Hence, the formalism is 
suitable for the study of nonequilibrium phenomena in lattice models. Inhomogeneous layered structures can be treated via a mapping to a coupled set of impurity problems, each representing one layer [20,21]. A detailed description of the formalism and of our numerical implementation is given in Ref. [18] for the translationally invariant case, and in Ref. [22] for the inhomogeneous DMFT formalism. To take into account antiferromagnetic ordering within and between the layers, we have implemented this formalism with a magnetic unit cell of two sites within the layers. Metallic leads with a flat density of states are included in the formalism by adding additional lead self-energies [22]. The impurity model is solved here using the perturbative hybridization expansion (noncrossing approximation, NCA) [23]. The validity of the NCA for the (photodoped) Mott insulating phase has been addressed previously for homogeneous bulk systems. NCA gives qualitatively the same results as higher order variants of the expansion for the current in the dielectric breakdown regime [24] and for the relaxation of a photo-excited Mott-insulator [25]. Simulations of inhomogeneous systems in an antiferromagnetic state are numerically considerably more demanding, such that higher-order schemes (one-crossing approximation) are not a realistic option at this time.

We compute the time evolution of various observables, in particular, the (layer-resolved) density $n(t, z)=$ $(1 / L) \sum_{j \sigma}\left\langle n_{j, z, \sigma}\right\rangle$ and staggered magnetization $m(t, z)=$ $(1 / L) \sum_{j} e^{i q j}\left\langle n_{j, z, \uparrow}-n_{j, z, \downarrow}\right\rangle$, with $\boldsymbol{q}=(\pi, \pi)$. The photoemission spectrum $I\left(\Omega, t_{p}, z\right)$ is obtained from a convolution of the lesser component of the local, layer-resolved Green's function $G_{z}^{<}\left(t, t^{\prime}\right)=i \sum_{\sigma}\left\langle c_{i z \sigma}^{\dagger}\left(t^{\prime}\right) c_{i z \sigma}(t)\right\rangle$ with the probe-pulse envelope $s(t)=\exp \left(-t^{2} / \delta t^{2}\right)$ [26],

$$
I\left(\Omega, t_{p}, z\right)=\int d t d t^{\prime} s\left(t-t_{p}\right) s\left(t^{\prime}-t_{p}\right) e^{i \Omega\left(t-t^{\prime}\right)} G_{z}^{<}\left(t, t^{\prime}\right) .
$$

The width $\delta t$ and its inverse $\hbar / \delta t$ determine the time and energy resolution, respectively. The spectrum also provides an unambiguous way to measure the density of photoexcited doublon and hole carriers, $n_{d}(t, z)$ and $n_{h}(t, z)$ : Physically, a doublon is a resonance that is well defined only on timescales much longer than that of virtual charge fluctuations, and, hence, $n_{d}(t, z)$ and $n_{h}(t, z)$ are given by the integrated photoemission weight in the upper Hubbard band and the integrated inverse photoemission weight in the lower Hubbard band, respectively. (In a nonequilibrium state with simultaneous excitation of doublons and holes, carrier densities cannot be computed from the density.)

Results.-We choose $\left|t^{\|}\right|=\left|t^{\perp}\right| \equiv t_{*}$ as the unit of energy, and take $U=20$, such that the system becomes a Mott insulator with a gap comparable to the free threedimensional bandwidth $12 t_{*}$. For a gap of $1 \mathrm{eV}$ [13], the unit of time is thus $\hbar / t_{*} \approx 5$ fs. In Figure 1(a) we plot the layer-resolved spectral functions of the heterostructure in equilibrium. All simulations are performed for un-biased leads. Up to $\Delta E \approx 2$, where the Hubbard bands at the boundary become degenerate with the Fermi energy $E_{F}=0$, the coupling to the noninteracting leads yields only a weak transfer of spectral weight into the gap region at the boundary layers, and slight hole or electron doping [Fig. 1(b)]. For $\Delta E \approx 1.5$, the ratio of bandwidth, gap, and $\Delta E$ is comparable to the LVO heterostructure studied in Ref. [13]. The system becomes antiferromagnetically ordered for temperatures below $T=T_{N} \approx 0.3$ [Fig. 1(c)].

Photoexcitation is simulated by a few-cycle in-plane electric field pulse centered at time $t_{0}=2 \pi / \Omega$ and frequency $\Omega=U, \boldsymbol{E}(t)=\left(E_{0}, E_{0}, 0\right) \sin (\Omega t) \exp [-4.605(t-$ $\left.\left.t_{0}\right)^{2} / t_{0}^{2}\right] \Theta\left(2 t_{0}-t\right) \Theta(t)$, taken into account by the Peierls substitution [22]. The broad frequency distribution of the short pulse can mimic an incoherent source like the sun. The pulse promptly creates a small density of holelike and electronlike carriers (doublons) in the Mott insulating structure. As expected for $U / t_{*} \gg 1$, we observe no recombination of photoexcited carriers within the time scale of our simulation [25,27]. The evolution of the doublon and hole densities $n_{d}(t, z)$ and $n_{h}(t, z)$ under

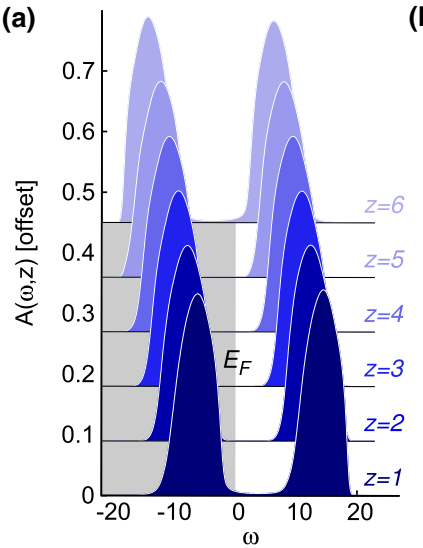

(d)

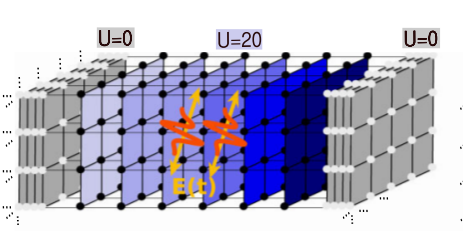

(b)

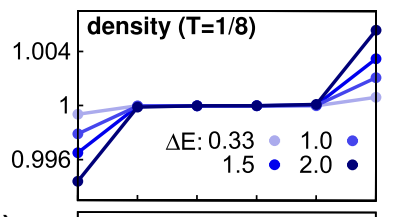

(c)

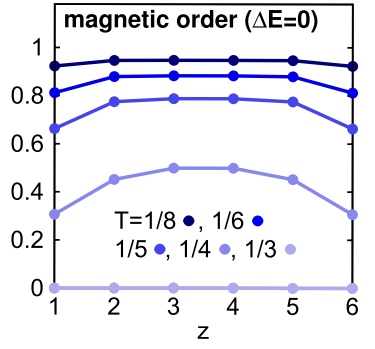

(e)

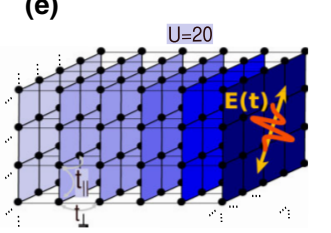

FIG. 1 (color online). Equilibrium configuration of the heterostructure and nonequilibrium setup. (a) Layer and frequency resolved spectral function $A(\omega, z)$ in the Mott heterostructure with leads, for temperature $T=1 / 3$ (paramagnetic phase) and internal field $\Delta E=1.5 ; \omega=0$ is the Fermi level of the external leads. (b) Layer-dependent density for various gradients $\Delta E$. (c) Layer-dependent antiferromagnetic order for various temperatures up to the Néel temperature. (d) Setup used to study the doublon and hole diffusion to the leads. (e) Setup without leads, which allows us to study the spreading of doublons over a longer distance. 
the influence of the internal field is fundamentally different in the paramagnetic and antiferromagnetic structures [Figs. 2(a)-(d)]: In the latter, doublons and holes rapidly separate and move to the boundary layers, where they are collected by the leads. This process becomes faster for larger $\Delta E$, as evidenced by the spatial mean $\bar{z}_{d / h}(t)=$ $\sum_{z} z n_{d / h}(z, t) / \sum_{z} n_{d / h}(z, t)$ of the distributions [dotted lines in Figs. 2(a)-(d)]. In the paramagnetic phase, on the other hand, we observe almost no separation of carriers beyond a polarization proportional to $\Delta E$, which builds up already during the excitation (offset between $\bar{z}_{d}$ and $\bar{z}_{h}$ ). The current $J_{\text {lead }}$ into the leads shows a similar tendency [Figs. 2(e)-(h)]. In the antiferromagnetic sample, it reaches its maximum roughly when $50 \%$ of the total number of
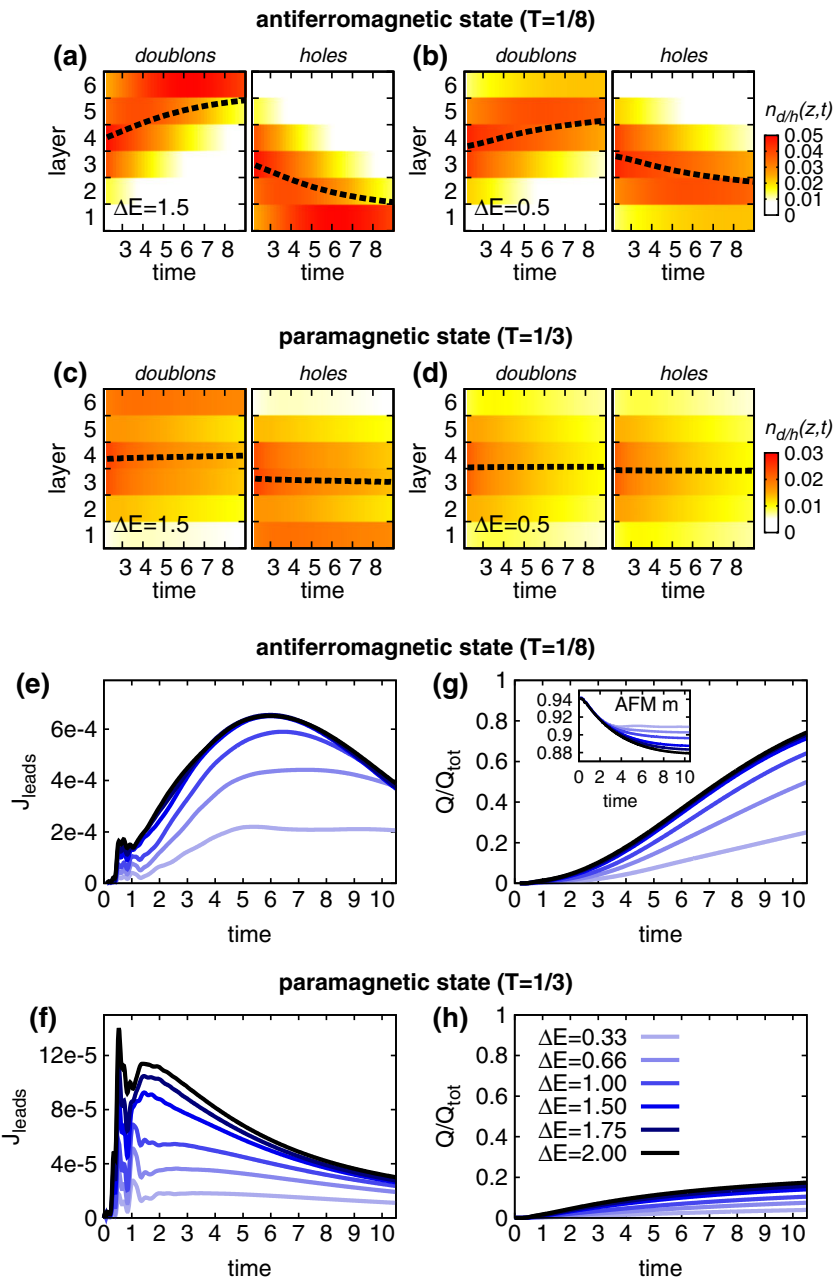

FIG. 2 (color online). (a)-(d) Time and layer-dependent doublon and hole densities for two different internal fields. The dashed lines indicate the mean $\bar{z}_{d / h}(t)$ of the distributions in space. The heterostructure is excited at layers $z=3$, 4. (e),(f) Current to the leads for various $\Delta E$ [see key in panel (h)]. Here, the heterostructure is excited at all layers. $(\mathrm{g}),(\mathrm{h})$ Time-integrated current $Q=\int_{0}^{t} d t^{\prime} J_{\text {lead }}\left(t^{\prime}\right)$, compared to the total photoexcited charge, for the same parameters as in (e) and (f). The inset in (g) shows the layer-averaged antiferromagnetic order parameter as a function of time. photoexcited carriers have tunneled into the leads, which takes only a few hopping times for large fields $\Delta E \gtrsim 1$. In the paramagnet, the current starts to decrease already shortly after the pulse, when most of the photocarriers still reside within the heterostructure. The simulations are limited to short times, but we can take the current at $t=10$ to estimate a lower bound for the time needed for the carriers to leave the structure. At $\Delta E=1$, this gives $t=150$, i.e., $0.75 \mathrm{ps}$ for a gap of $1 \mathrm{eV}$. On picosecond time scales, however, weak interactions such as couplings to optical phonons or nonlocal Coulomb interactions not included in our model Hamiltonian may lead to exciton formation and recombination [15,28], which would degrade the efficiency of the device.

To explain the striking difference between paramagnetic and antiferromagnetic samples, we first note that the decaying current in the paramagnetic sample resembles the behavior of isolated (nonintegrable) metals $[9,29,30]$ and Mott insulators [24]. In fact, to separate doublons and holes by one layer, the charges must release an energy proportional to $\Delta E$, because the electronic bandwidth is finite, but heat transport without particle transport or magnons is slow. In the antiferromagnetic phase, on the other hand, flipping spins provides a fast channel to release this energy locally. Indirect evidence for an energy transfer to the spin sector is given by the decrease of the magnetization $m$ with time [inset in Fig. 2(g)]. Furthermore, we find that the drift velocity $v_{d}(t)=d / d t \bar{z}_{d}(t)$ increases with $m^{2}$ as the temperature is decreased and the magnetic order strengthens (Fig. 3). Such a behavior may be expected because for each hopping the average number of spin flips
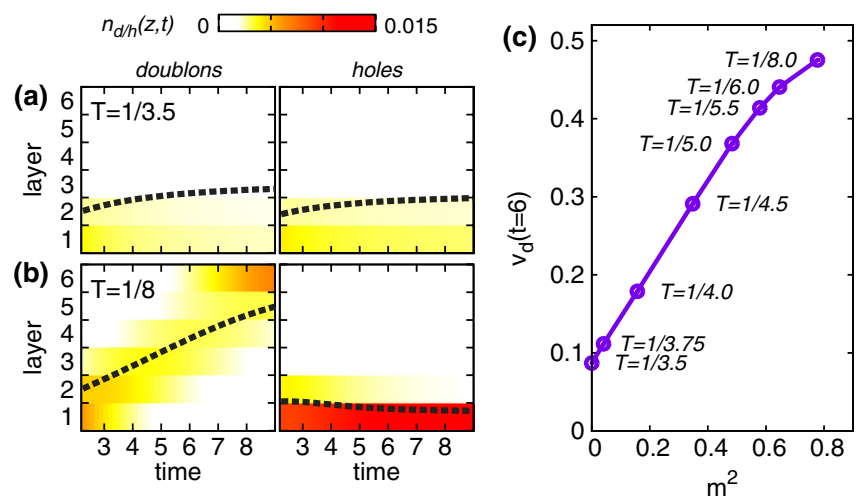

FIG. 3 (color online). Temperature dependence of carrier motion. (a),(b) Layer and time-resolved doublon and hole densities for two temperatures, $T=1 / 3.5$ (paramagnetic) and $T=1 / 8$ (antiferromagnetic). To study the carrier motion over a longer distance, we consider a heterostructure which is not coupled to leads, apply the excitation at layer $z=1$, and focus on the doublon dynamics (holes remain trapped at the boundary); see sketch in Fig. 1(e). The internal field is $\Delta E=1$. The dotted line shows the spatial mean $\bar{z}_{d / h}(t)$. (c) Velocity $v_{d}(t)=$ $d \bar{z}_{d}(t) / d t$ at intermediate time $t=6$, plotted for various temperatures against the square $m^{2}$ of the layer-averaged staggered magnetization. 
antiferromagnetic state $(T=1 / 8)$

paramagnetic state $(T=1 / 3)$

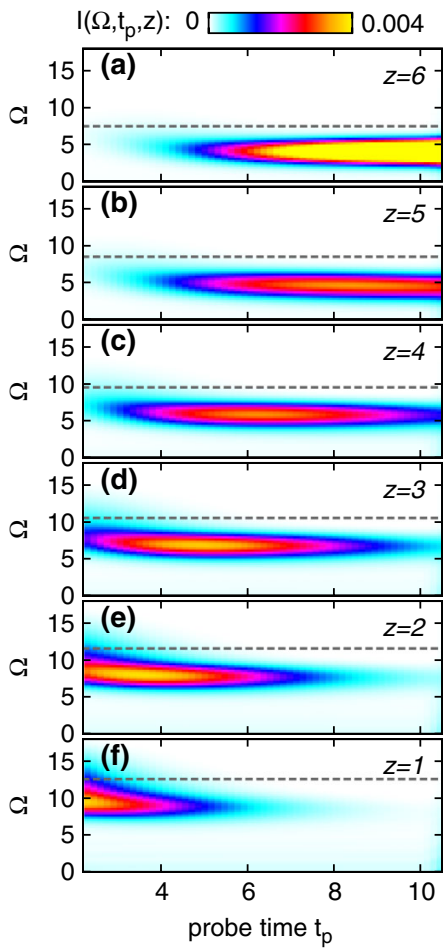

$\mathrm{I}\left(\Omega, \mathrm{t}_{\mathrm{p}}, \mathrm{z}\right): 0 \square 0.002$

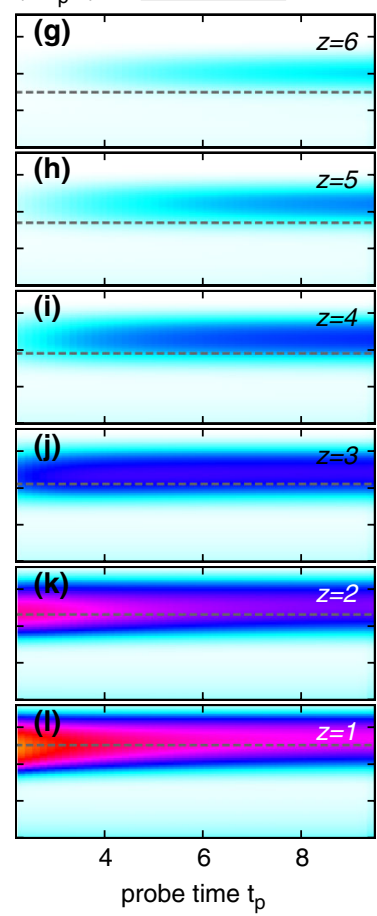

FIG. 4 (color online). Layer-resolved, spin-integrated photoemission spectrum $I\left(\Omega, t_{p}, z\right)$ of the upper Hubbard band, for the same geometry as in Fig. 3. (a)-(f) Antiferromagnetic structure at $T=1 / 8$. (g)-(l) Paramagnetic structure at $T=1 / 3$. The dashed lines indicate the center of the upper Hubbard band.

against the antiferromagnetic exchange $J=4 t_{*}^{2} / U$ is proportional to $m$, and, hence, the maximal rate of change of the spin energy $\mathrm{Jm}^{2}$ is proportional to $\mathrm{m}^{2}$ and a hopping rate.

A more detailed picture of the carrier dynamics can be obtained from the photoemission spectrum $I\left(\Omega, t_{p}, z\right)$ (occupied density of states) as a function of layer, probe time $t_{p}$, and frequency (Fig. 4). We computed $I\left(\Omega, t_{p}, z\right)$ with a time resolution of $\delta t=1$ (set by the probe duration), and an uncertainty-limited frequency resolution $\delta \omega=\hbar / \delta t$ (see Methods section). In the antiferromagnetic case, the initially broad energy distribution in the upper Hubbard band of the excited layer $(z=1)$ rapidly accumulates at the lower band edge, which demonstrates the transfer of the kinetic energy of photocarriers to the spin background. This cooling mechanism is absent in the paramagnetic phase, and, consequently, there is no significant redistribution of spectral weight within the band, as it has also been observed in homogeneous Mott systems [25,31]. As the carriers gradually move towards the other boundary, the spectral weight in the antiferromagnetic structure further accumulates in the lower half of the Hubbard band, while it remains pinned at the same energy in the paramagnetic calculation (thus shifting upwards within the Hubbard band layer by layer). antiferromagnetic state $(T=1 / 8)$
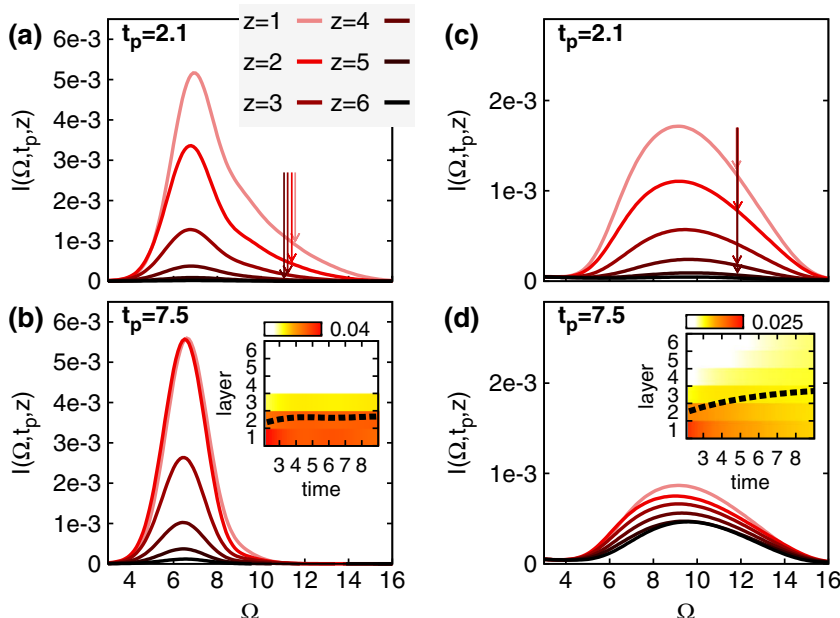

FIG. 5 (color online). Diffusion of carriers in zero field: Photoemission spectrum of the upper Hubbard band shortly after the excitation $\left(t_{p}=2.1\right)$ and at time $t_{p}=7.5$. The setup is identical to Fig. 3, but for $\Delta E=0$. (a) $t_{p}=2.1, T=1 / 8$. (b) $t_{p}=7.5, T=1 / 8$. (c) $t_{p}=2.1, T=1 / 3$. (d) $t_{p}=7.5$, $T=1 / 3$. Vertical arrows in (a) and (c) indicate the mean of the distribution in the spectral region $10<\omega<16$. Insets in (b) and $(\mathrm{d})$ : density $n_{d}(z, t)$ for the respective temperatures. In zero field, the result for $n_{h}(z, t)$ is identical.

Interestingly, the opposite effect is observed for the pure diffusion of carriers at $\Delta E=0$ [10]. The diffusion of carriers in the antiferromagnetic phase perturbs the magnetic order and thus implies an exchange energy cost. In equilibrium, this leads to the formation of heavy spin polarons, which are visible in the low-energy part of the spectrum [32-34]. After photoexcitation, carriers rapidly exhaust their kinetic energy to overcome the exchange energy cost, the spectral occupation accumulates at the lower edge of the upper Hubbard band, and further diffusion relies on much slower energy transport processes [Figs. 5(a), (b)]. The high-energy tails of the distributions at early times are layer dependent, which reveals the energy loss of carriers as they move from layer to layer. In the paramagnet, on the other hand, doublons and holes spread throughout the sample in a purely diffusive manner, and the distribution functions remain broad at all layers and times [Figs. 5(c), (d)].

In summary, our results demonstrate the decisive role of spin flip scattering for the carrier dynamics in Mott heterostructures with antiferromagnetic correlations. The spin background can act as a buffer that absorbs energy on femtosecond time scales, and thus enables a rapid carrier separation in photovoltaic devices (the $\mathrm{LaVO}_{3}$ system proposed in Ref. [13] is indeed magnetically ordered [35]). Phonons might play a similar role, but for typical parameters the coupling to spins is expected to be dominant [8]. In a broader context, exploiting the spin scattering and its ability to rapidly cool injected carriers may become an 
important design principle for devices which operate on a femtosecond time scale. It can boost the transport of carriers in the strong field gradients of polar heterostructures, or significantly delay the spreading of carriers in zero field. Conversely, inhomogeneous setups may be used to probe the ultrafast exchange of energy between particles and spins: While optical excitations typically penetrate many atomic layers, photoemission can be made surface sensitive. In a heterostructure with band bending towards the surface, one can thus expect a delay between the maximum photoemission signal and the pump, which reflects the spreading of the carriers.

We thank J. Bonča, K. Held, D. Golež, Z. Lenarčič, T. Oka, P. Prelovśek, G. Sangiovanni, N. Tsuji, and L. Vidmar for fruitful discussions. The calculations were run on the supercomputer HLRN-II of the North-German Supercomputing Alliance. P.W. acknowledges support from FP7/ERC starting Grant No. 278023.

*martin.eckstein@mpsd.cfel.de

[1] A. Ohtomo, D. A. Muller, J. L. Grazul, and H. Y. Hwang, Nature (London) 419, 378 (2002).

[2] A. Ohtomo and H. Y. Hwang, Nature (London) 427, 423 (2004).

[3] S. Okamoto and A. J. Millis, Nature (London) 428, 630 (2004).

[4] D. Fausti, R. I. Tobey, N. Dean, S. Kaiser, A. Dienst, M. C. Hoffmann, S. Pyon, T. Takayama, H. Takagi, and A. Cavalleri, Science 331, 189 (2011).

[5] A. Kirilyuk, A. V. Kimel, and T. Rasing, Rev. Mod. Phys. 82, 2731 (2010).

[6] A. D. Caviglia et al., Phys. Rev. Lett. 108, 136801 (2012).

[7] M. Mierzejewski, L. Vidmar, J. Bonča, and P. Prelovšek, Phys. Rev. Lett. 106, 196401 (2011).

[8] L. Vidmar, J. Bonča, T. Tohyama, and S. Maekawa, Phys. Rev. Lett. 107, 246404 (2011).

[9] A. Amaricci, C. Weber, M. Capone, and G. Kotliar, Phys. Rev. B 86, 085110 (2012).

[10] D. Golež, J. Bonča, M. Mierzejewski, and L. Vidmar, Phys. Rev. B 89, 165118 (2014).

[11] N. Nakagawa, H. Y. Hwang, and D. A. Muller, Nat. Mater. 5, 204 (2006).
[12] G. Singh-Bhalla, C. Bell, J. Ravichandran, W. Siemons, Y. Hikita, S. Salahuddin, A. F. Hebard, H. Y. Hwang, and R. Ramesh, Nat. Phys. 7, 80 (2011).

[13] E. Assmann, P. Blaha, R. Laskowski, K. Held, S. Okamoto, and G. Sangiovanni, Phys. Rev. Lett. 110, 078701 (2013).

[14] H. Okamoto, T. Miyagoe, K. Kobayashi, H. Uemura, H. Nishioka, H. Matsuzaki, A. Sawa, and Y. Tokura, Phys. Rev. B 82, 060513 (2010).

[15] M. Mitrano et al., Phys. Rev. Lett. 112, 117801 (2014).

[16] S. Dal Conte et al., Science 335, 1600 (2012).

[17] J. K. Freericks, V. M. Turkowski, and V. Zlatić, Phys. Rev. Lett. 97, 266408 (2006).

[18] H. Aoki, N. Tsuji, M. Eckstein, M. Kollar, T. Oka, and P. Werner, Rev. Mod. Phys. 86, 779 (2014).

[19] A. Georges, G. Kotliar, W. Krauth, and M. J. Rozenberg, Rev. Mod. Phys. 68, 13 (1996).

[20] M. Potthoff and W. Nolting, Phys. Rev. B 59, 2549 (1999).

[21] J. K. Freericks, Phys. Rev. B 70, 195342 (2004).

[22] M. Eckstein and P. Werner, Phys. Rev. B 88, 075135 (2013).

[23] M. Eckstein and P. Werner, Phys. Rev. B 82, 115115 (2010).

[24] M. Eckstein, T. Oka, and P. Werner, Phys. Rev. Lett. 105, 146404 (2010).

[25] M. Eckstein and P. Werner, Phys. Rev. B 84, 035122 (2011).

[26] J. K. Freericks, H. R. Krishnamurthy, and T. Pruschke, Phys. Rev. Lett. 102, 136401 (2009).

[27] R. Sensarma, D. Pekker, E. Altman, E. Demler, N. Strohmaier, D. Greif, R. Jördens, L. Tarruell, H. Moritz, and T. Esslinger, Phys. Rev. B 82, 224302 (2010).

[28] Z. Lenarčič and P. Prelovšek, Phys. Rev. Lett. 111, 016401 (2013).

[29] M. Mierzejewski and P. Prelovšek, Phys. Rev. Lett. 105, 186405 (2010).

[30] M. Eckstein and P. Werner, Phys. Rev. Lett. 107, 186406 (2011).

[31] B. Moritz, A. F. Kemper, M. Sentef, T. P. Devereaux, and J. K. Freericks, Phys. Rev. Lett. 111, 077401 (2013).

[32] R. Strack and D. Vollhardt, Phys. Rev. B 46, 13852 (1992).

[33] G. Sangiovanni, A. Toschi, E. Koch, K. Held, M. Capone, C. Castellani, O. Gunnarsson, S.-K. Mo, J. W. Allen, H.-D. Kim, A. Sekiyama, A. Yamasaki, S. Suga, and P. Metcalf, Phys. Rev. B 73, 205121 (2006).

[34] C. Taranto, G. Sangiovanni, K. Held, M. Capone, A. Georges, and A. Toschi, Phys. Rev. B 85, 085124 (2012).

[35] Although the material consists of ferromagnetically coupled antiferromagnetic layers, photocarriers can still perturb the spin background through hoppings within the planes and thus rapidly dissipate their kinetic energy. 